\title{
Abolition of Spontaneous Rhythmic Contractions of Isolated Monkey Coronary Arteries by Diltiazem, Nifedipine, Verapamil and Nicorandil but Not by Nitroglycerin
}

\author{
Kuniaki Ishit, Teruyuki Yanagisawa, Keisuke Satoh \\ and Norio TaIra \\ Department of Pharmacology, Tohoku University School of \\ Medicine, Sendai 980
}

\begin{abstract}
Ishit, K., Yanagisawa, T., Satoh, K. and Taira, N. Abolition of Spontaneous Rhythmic Contractions of Isolated Monkey Coronary Arteries by Diltiazem, Nifedipine, Verapamil and Nicorandil but Not by Nitroglycerin. Tohoku J. exp. Med., 1985, 145 (1), 85-90 — Three of 12 coronary artery rings obtained from one of 9 rhesus monkeys (Macaca mulatta) (113 rings in all) and 4 of 12 coronary artery rings obtained from one of 2 patas monkeys (Erythrocebus patas patas) (28 rings in all) developed spontaneous rhythmic contractions. In 5 of these 7 coronary artery rings, rhythmic contractions occurred long enough to be subjected to examinations of drug effects. In these preparations the effects of the calcium entry blockers, diltiazem, nifedipine and verapamil, and nicorandil, a nitrate vasodilator thought to produce vasodilatation by an increase in membrane potassium permeability, and nitroglycerin were investigated on rhythmic contractions. Diltiazem $\left(10^{-7}\right.$ and $\left.10^{-6} \mathrm{M}\right)$, nifedipine $\left(10^{-8}\right.$ and $\left.10^{-7} \mathrm{M}\right)$, verapamil $\left(10^{-7}\right.$ and $\left.10^{-6} \mathrm{M}\right)$ and nicorandil $\left(10^{-5}\right.$ and $\left.10^{-4} \mathrm{M}\right)$ suppressed rhythmic contractions in a concentration-dependent manner, but nitroglycerin $\left(10^{-7}-10^{-5} \mathrm{M}\right)$ was entirely ineffective. The results were discussed in relation to the mechanisms underlying spontaneous rhythmic contractions of coronary arteries and their role in vasospastic angina pectoris. coronary artery ; coronary spasm ; monkey ; calcium antagonist; nitrate
\end{abstract}

Spontaneous rhythmic contractions have been observed in isolated coronary arteries obtained from postmortem human hearts (Golenhofen 1978; Kawasaki et al. 1981) or from patients undergoing cardiac transplantation (Ross et al. 1980). This phenomenon is thought by some investigators to be related to coronary artery spasm associated with vasospastic angina pectoris (Ross et al. 1980 ; Kawasaki et al. 1981). Spontaneous rhythmic contractions are abolished in calcium free solutions (Ross et al. 1980) or by the calcium entry blockers, nifedipine (Golenhofen 1978) and verapamil (Ross et al. 1980). Thus, it has been suggested that calcium influx would be responsible for spontaneous rhythmic contractions (Ross

Received for publication, May 9, 1984. 
et al. 1980). In the course of experiments to investigate the responsiveness of isolated monkey coronary arteries to a variety of agonists we encountered spontaneous rhythmic contractions. Since spontaneous rhythmic contractions are rare phenomena even in monkey coronary arteries as described below and have never been found in dog coronary arteries, it would be of value to report even limited observations of them and of drug effects thereon.

\section{Materials and Methods}

Nine rhesus monkeys (Macaca mulatta) of either sex weighing 7-10 kg and 2 male patas monkeys (Erythrocebus patas patas) weighing $10 \mathrm{~kg}$ were anesthetized initially with ketamine hydrochloride (Ketalar ${ }^{\circledR}$, Sankyo) $(10 \mathrm{mg} / \mathrm{kg}$ i.m.) and subsequently with pentobarbital sodium (10-20 mg/kg i.v.), and given heparin sodium (1,000 units/kg i.v.). After the animals had been exsanguinated the hearts were rapidly excised. Coronary arteries were dissected out from the hearts and cleaned of fat and connective tissues in Krebs-Henseleit solution. The composition of the solution in $\mathrm{mM}$ was as follows: $\mathrm{NaCl}$ 118; KCl $4.7 ; \mathrm{CaCl}_{2} 2.55 ; \mathrm{MgSO}_{4}$ 1.18; $\mathrm{NaHCO}_{3} 24.9$; Glucose 11.1. The isolated arteries were cut into rings of $2 \mathrm{~mm}$ in width and the rings were suspended with resting tension of $0.5-1 \mathrm{~g}$ in organ baths containing $20 \mathrm{ml}$ of the solution. The temperature of the baths was maintained at $37^{\circ} \mathrm{C}$ and the solution was equilibrated with $95 \% \mathrm{O}_{2}$ and $5 \% \mathrm{CO}_{2}$. Tension developed by coronary artery rings was measured with strain-gauge transducers (Shinko, UL-10) and recorded on a chart with recorders (Toa Denpa, FBR-252A). The preparations were equilibrated at least for $1 \mathrm{hr}$ before exposure to agonists such as acetylcholine and methoxamine or a high potassium solution. The high potassium condition was made by addition of $90 \mathrm{mM} \mathrm{KCl}$ to the normal solution. In the preparations which developed spontaneous rhythmic contractions the effects of diltiazem, nifedipine, verapamil, nicorandil and nitroglycerin were investigated.

The drugs used were acetylcholine chloride (Daiichi Seiyaku), methoxamine hydrochloride $(10 \mathrm{mg} / \mathrm{ml}$ in ampule, Nippon Shinyaku), diltiazem hydrochloride (Tanabe Seiyaku), nifedipine $(100 \mu \mathrm{g} / \mathrm{ml}$ in ampule, Bayer), verapamil hydrochloride (Knoll), nicorandil (Chugai Pharmaceutical) and nitroglycerin $(617 \mu \mathrm{g} / \mathrm{ml}$ of distilled water in ampule, Nippon Kayaku). Nifedipine, nitroglycerin and methoxamine solutions in ampules were diluted with distilled water to the desired concentrations and other drugs were dissolved in distilled water. All drug solutions in a constant volume of $0.1 \mathrm{ml}$ were added directly to the bathing media. A single drug was administered in a cumulative manner. After an experiment on a single drug, the preparation was washed 1-5 times and equilibrated at least for $1 \mathrm{hr}$ before the start of experiment on another drug.

\section{Results}

\section{Spontaneous rhythmic contractions}

Spontaneous rhytrhmic contractions occurred in 3 (the left circumflex coronary artery, inner diameter: $0.6-0.8 \mathrm{~mm}$; outer diameter: $1.4-1.5 \mathrm{~mm}$ ) of 12 coronary artery rings of one of 9 rhesus monkeys (113 rings in all) and 4 (the left circumflex coronary artery, inner diameter : $0.5-0.8 \mathrm{~mm}$; outer diameter : $1.0-1.5$ $\mathrm{mm}$ ) of 12 coronary artery rings of one of 2 patas monkeys (28 rings in all). Rhythmic contractions emerged spontaneously during equilibration or exposure to methoxamine, or after washout of acetylcholine or the high potassium solution. Durations and interval of rhythmic contractions varied depending upon prepara- 
tions; durations of single contractions ranged from 13 to $60 \mathrm{~min}$ and intervals ranged from 12 to $100 \mathrm{~min}$. However, for each preparation variations in the durations and intervals were rather small.

\section{Effects of drugs}

In 5 of the 7 coronary artery rings ( 2 rings from one rhesus monkey and 3 rings from one patas monkey) spontaneous rhythmic contractions occurred for periods long enough to be sujected to examinations of drug effect. Fig. 1 shows the effects of diltiazem, nifedipine, nicorandil and nitroglycerin on spontaneous rhythmic contractions of one of the 2 rings from the rhesus monkey. In this preparation contractions amounting to 2.1 to $5.7 \mathrm{~g}$ at peak values and lasting 13 to $24 \mathrm{~min}$ recurred spontaneously at intervals of 12 to $24 \mathrm{~min}$ for more than $24 \mathrm{hr}$. Under these conditions diltiazem $\left(10^{-7}-10^{-6} \mathrm{M}\right)$, nifedipine $\left(10^{-9}-10^{-7} \mathrm{M}\right)$ and nicorandil $\left(10^{-6}-10^{-4} \mathrm{M}\right)$ suppressed spontaneous contractions in a concentration-dependent manner and abolished them in the respective highest concentrations. In contrast, nitroglycerin in concentrations up to $10^{-5} \mathrm{M}$ was ineffective. Essentially similar results were obtained with nifedipine in the other preparation from the rhesus monkey and 2 preparations from the patas monkey; in the preparation from the rhesus monkey $10^{-8} \mathrm{M}$ nifedipine abolished the contractions and in the 2 preparations from the patas monkey $10^{-7} \mathrm{M}$ nifedipine abolished the contractions. Nitroglycerin $\left(10^{-7}-10^{-5} \mathrm{M}\right)$ was also ineffetive in the preparation of the rhesus monkey in which $10^{-8} \mathrm{M}$ nifedipine abolished the spontaneous contractions. Verapamil $\left(10^{-7}\right.$ and $\left.10^{-6} \mathrm{M}\right)$ suppressed spontaneous rhythmic contractions and
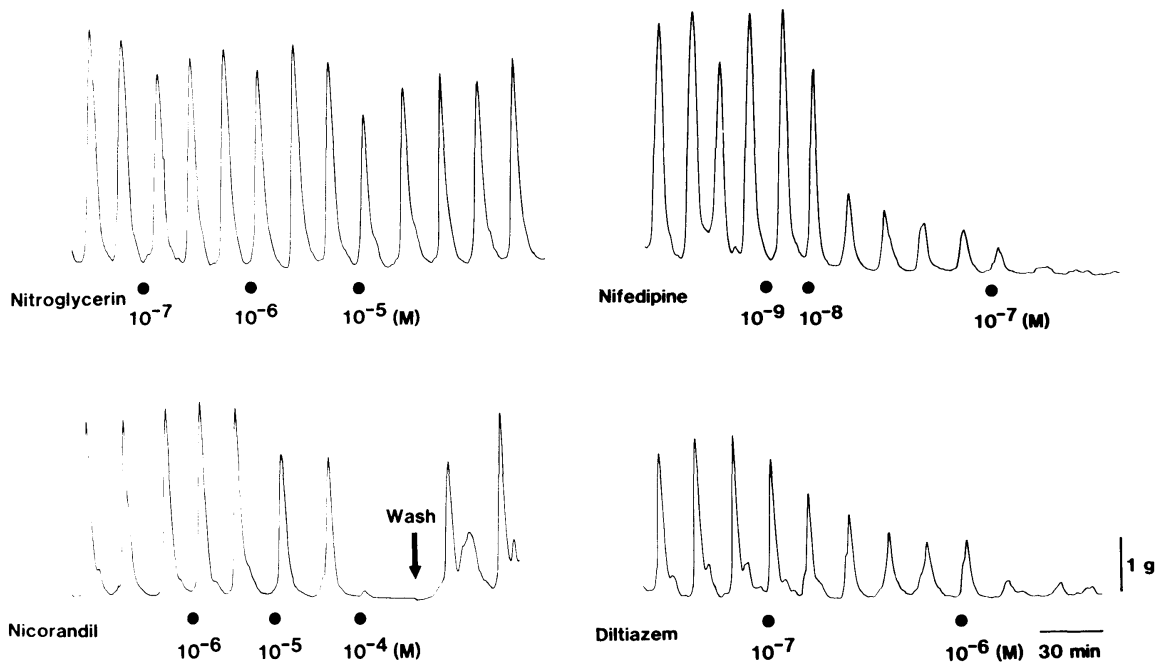

Fig. 1. Spontaneous rhythmic contractions of a coronary artery ring of the left circumflex coronary artery of a rhesus monkey and effects of vasodilators thereon. Experiments on this preparation ran from the left to the right in the upper panel and then in a similar way in the lower panel. Drugs were applied at dots. 
abolished them in the higher concentration in the remaining one preparation from the patas monkey only in which the effect of verapamil was examined.

\section{Discussion}

In the present experiments diltiazem, nifedipine and verapamil were all capable of suppressing spontaneous rhythmic contractions of coronary arteries isolated from two species of subhuman primates. The present results are similar to the findings of the previous investigators that spontaneous rhythmic contractions of isolated human coronary arteries were susceptible to the blocking action of calcium entry blockers such as nifedipine (Golenhofen 1978) and verapamil (Ross et al. 1980), suggesting that an increased calcium influx plays an important role in the development of spontaneous rhythmic contractions. The present results that nitroglycerin was ineffective in suppressing spontaneous rhythmic contractions are at variance with the findings by Ross et al. (1980) that spontaneous rhythmic contractions of isolated human coronary arteries were suppressed by nitroglycerin. However, the present results are consistent with the findings by Golenhofen (1978) that spontaneous rhythmic contractions of isolated human coronary arteries were more resistant to nitroglycerin than to the calcium entry blocker, nifedipine.

The effectiveness of the calcium entry blockers, nifedipine, diltiazem and verapamil, and the ineffectiveness of nitroglycerin in suppressing spontaneous rhythmic contractions observed in the present experiments are also consistent with those observed in in vivo experiments on a Japanese monkey (Taira et al. 1983). In this monkey oscillatory changes in blood flow through the left anterior descending coronary artery produced by intra-arterial infusion of acetylcholine were suppressed by diltiazem and nifedipine but not by nitroglycerin. Strictly speaking oscillatory changes in coronary blood flow observed in vivo in the Japanese monkey were different from spontaneous rhythmic contractions of isolated coronary arteries in duration of single changes and intervals between two changes. Nevertheless, similar drug susceptibility and resistance of the two phenomena imply that they share a common mechanism.

As opposed to the ineffectiveness of nitroglycerin, nicorandil was effective in suppressing spontaneous rhythmic contractions in the present experiments. In this respect nicorandil differs from nitroglycerin, although nicorandil is a nitrate and shares a property with nitroglycerin to increase intracellular levels of cyclic GMP in vascular smooth muscle (Endoh and Taira 1983; Holzmann 1983). Nicorandil is thought to produce relaxation of coronary arterial smooth muscle mainly by increasing membrane potassium permeability leading to hyperpolarization (Furukawa et al. 1981). When the membrane potential is hyperpolarized, the voltage-dependent calcium channel in coronary arterial smooth muscle will become less likely to open. By this mechanism intracellular free calcium will be reduced. In other words, nicorandil appears to behave as an indirectly-acting 
calcium entry blocker. Taken together this with the effectiveness of the directlyacting calcium entry blockers, an increased calcium influx plays a key role in the spontaneous rhythmic contractions of isolated subhuman primate coronary arteries and also of isolated human coronary arteries. However, the ineffectiveness of nitroglycerin on these spontaneous rhythmic contractions casts a doubt on the concept that they have something to do with coronary artery spasm, because nitroglycerin is highly efficacious in releasing coronary artery spasm associated with vasospastic angina (Dhurandhar et al. 1972 ; Oliva et al. 1973).

Finally the question will be raised why spontaneous rhythmic contractions developed in coronary arteries of a very small number of monkeys and in a limited number of coronary artery rings obtained from the same coronary artery. A similar question will be directed to cardiologists why coronary artery spasm occurs only in particular individuals. These questions are not answered at the moment.

\section{Acknowledgments}

This study was supported by a Grant-in-Aid for Scientific Research (No. 57440030) from the Ministry of Education, Science and Culture, Japan. We gratefully acknowlege the generous supply of nine rhesus monkeys and two patas monkeys by Mr. Shigetaka Kotera, Zoo Director, Primate Zoo, Japan Monkey Centre, Inuyama. We are also grateful to Bayer Yakuhin Co., Ltd., Osaka for ampules of nifedipine, to Chugai Pharmaceutical Co., Ltd., Tokyo for nicorandil, to Eisai Co., Ltd., Tokyo for verapamil, Nippon Kayaku Co., Ltd., Tokyo for nitroglycerin, and to Tanabe Seiyaku Co., Ltd., Osaka for diltiazem.

\section{References}

1) Dhurandhar, R.W., Watt, D.L., Silver, M.D., Trimble, A.S. \& Adelman, A.G. (1972) Prinzmetal's variant form of angina with arteriographic evidence of coronary arterial spasm. Amer. J. Cardiol., 30, 902-905.

2) Endoh, M. \& Taira, N. (1983) Relationship between relaxation and cyclic GMP formation caused by nicorandil in canine mesenteric artery. Naunyn-Schmiedeberg's Arch. Pharmacol., 322, 319-321.

3) Furukawa, K., Itoh, T., Kajiwara, M., Kitamura, K., Suzuki, H., Ito, Y. \& Kuriyama, H. (1981) Vasodilating actions of 2-nicotinamidoethyl nitrate on porcine and quineapig coronary arteries. J. Pharmacol. exp. Ther., 218, 248-259.

4) Golenhofen, K. (1978) Activation mechanisms in smooth muscle of human coronary arteries and their selective inhibition. Naunyn-Schmiedeberg's Arch. Pharmacol., 302, R36.

5) Holzmann, S. (1983) Cyclic GMP as possible mediator of coronary arterial relaxation by nicorandil (SG-75) J. cardiovasc. Pharmacol., 5, 364-370.

6) Kawasaki, K., Seki, K. \& Hosoda, S. (1981) Spontaneous rhythmic contractions in isolated human coronary arteries. Experientia, 37, 1291-1292.

7) Oliva, P.B., Potts, D.E. \& Pluss, R.G. (1973) Coronary arterial spasm in Prinzmetal angina. Documentation by coronary arteriography. New Engl. J. Med., 288, 745751.

8) Ross, G., Stinson, E., Schroeder, J. \& Ginsburg, R. (1980) Spontaneous phasic activity of isolated human coronary arteries. Cardiovasc. Res., 14, 613-618.

9) Taira, N., Satoh, K., Maruyama, M. \& Yamashita, S. (1983) Sustained coronary 
constriction and its antagonism by calcium-blocking agents in monkeys and baboons. Circulat. Res., 52, Suppl. I. 40-46. 